\title{
Incidence Rates, Risk Factors, and Mortality Rates of Ventilator-Associated Pneumonia: A systematic Review and Meta-analysis
}

\section{CURRENT STATUS: UNDER REVIEW}

BMC Infectious Diseases $\triangle$ BMC Series

Haixia Wang

Shandong University

$\checkmark$ whx1346868869@gmail.comCorresponding Author

ORCiD: https://orcid.org/0000-0002-0403-7797

Mo Yi

Shandong University

Yuanmin Jia

Shandong University

Chenxiao Bai

Shandong University

Di Jiang

Shandong University

Yizhang Li

Shandong University

Ou Chen

Shandong University

DOI:

$10.21203 / \mathrm{rs} .2 .20681 / \mathrm{v} 1$

SUBJECT AREAS

Infectious Diseases

KEYWORDS

Ventilator-associated pneumonia, Incidence, Risk factors, Mortality 
Abstract

Background

Ventilator-associated pneumonia (VAP) is the second most common nosocomial infection in Intensive care units (ICUs) and is associated with the significant utilization of health-care resources. However the incidence rates, risk factors and mortality rates of VAP are controversial.

\section{Methods}

The Web of Science, PubMed and Cochrane Library databases through August 2019 were independently searched by two researchers to collect all relevant studies. Data including morbidity, risk factors and mortality rates of VAP were extracted for the systematic review and meta-analysis.

\section{Results}

Sixteen studies, including 4 case control studies and 12 cohort studies, on VAP were included in our analysis. In the meta-analysis, we found that the VAP rate was $22 / 1000$ ventilator-days $(95 \% \mathrm{Cl} 18$ 25\%). The data demonstrated that $\mathrm{H} 2$ blocker use (odds ratio (OR) 1.66; 95\% Cl 1.17-2.36),

nasogastric tube placement ( $\mathrm{OR} 2.88 ; 95 \% \mathrm{Cl}$ 1.03-8.04), enteral feeding (OR 3.02; 95\% Cl 2.18-4.20), central vascular catheter (CVC) placement (OR 3.84; 95\% Cl 2.55-5.78) and tracheotomy (OR 9.13; $95 \% \mathrm{Cl}$ 6.53-12.75) were risk factors of VAP. VAP was associated with an increased risk of mortality (OR 1.15; 95\% $\mathrm{Cl} 0.96-1.37$ ). Four articles that were not included in the meta-analysis indicated that hyperoxemia, increased intra-abdominal pressure, and genetic polymorphism may be risk factors for VAP.

\section{Conclusions}

This study may contribute to the development of improved infection control strategies for high-risk patients. Additional studies are needed in the future to clarify the incidence rates, risk factors and mortality rates of VAP.

Full Text

Due to technical limitations, full-text HTML conversion of this manuscript could not be completed. However, the manuscript can be downloaded and accessed as a PDF. 